\title{
Boundary regularity for elliptic systems with supquadratic growth condition
}

\section{Shuhong Chen ${ }^{1 *}$ and Zhong Tan ${ }^{2}$}

${ }^{*}$ Correspondence: shiny0320@163.com

'Department of Mathematics and Information Science, Zhangzhou Normal University, Zhangzhou, Fujian 363000, China

Full list of author information is available at the end of the article

\begin{abstract}
We consider boundary regularity for weak solutions of quasilinear elliptic systems with the supquadratic growth condition and obtain a general criterion for a weak solution to be regular in the neighborhood of a given boundary point. By an element covering argument combined with existing interior partial regularity results, we establish the boundary regularity result.
\end{abstract}

Keywords: quasilinear elliptic systems; A-harmonic approximation technique; boundary partial regularity

\section{Introduction}

In this paper, we consider the partial regularity for weak solutions of elliptic systems

$$
-D_{\alpha}\left(A_{i j}^{\alpha \beta}(x, u) D_{\beta} u^{j}\right)=B_{i}(x, u, D u), \quad x \in \Omega,
$$

where $\Omega$ is a bounded domain in $R^{n}$ with a boundary of class $C^{1}, n \geq 2, u$ and $B_{i}$ take values in $R^{N}, N>1$. Each $A_{i j}^{\alpha \beta}$ maps $\Omega \times R^{N}$ into $R$, and each $B_{i}$ maps $\Omega \times R^{N} \times R^{n N}$ into $R$. In theory partial regularity to (1.1) needs a priori existence of weak solutions. In order to define weak solutions to (1.1), one needs to impose certain structural and regularity conditions on $A_{i j}^{\alpha \beta}$ and the inhomogeneity $B_{i}$ as follows. For $m>2$,

(H1) There exists $L>0$ such that

$$
A_{i j}^{\alpha \beta}(x, \xi)(\nu, \tilde{v}) \leq L\left(1+|\xi|^{2}\right)^{\frac{m-2}{2}}|\nu||\tilde{v}| \quad \text { for all }(x, \xi,) \in \bar{\Omega} \times R^{N}, v, \tilde{v} \in R^{n N}
$$

(H2) $A_{i j}^{\alpha \beta}(x, \xi)$ is uniformly strongly elliptic, that is, for some $\lambda>0$, we have

$$
A_{i j}^{\alpha \beta}(x, \xi)(v, v) \geq \lambda\left(1+|\xi|^{2}\right)^{\frac{m-2}{2}}|v|^{2} \quad \text { for all }(x, \xi) \in \bar{\Omega} \times R^{N}, v \in R^{n N} .
$$

(H3) There exists a monotone nondecreasing concave function $\omega(s):[0, \infty) \rightarrow[0, \infty)$ with $\omega(0)=0$, continuous at 0 , such that for all $x, y \in \Omega, u, v \in R^{N}$,

$$
\left|A_{i j}^{\alpha \beta}(x, u)-A_{i j}^{\alpha \beta}(y, v)\right| \leq \omega\left(|x-y|^{m}+|u-v|^{m}\right) .
$$

(H4) The inhomogeneity satisfies the following growth condition:

$$
\left|B_{i}(x, \xi, \nu)\right| \leq\left(1+|\xi|^{2}\right)^{\frac{m-2}{2}} Q(x),
$$

o 2012 Chen and Tan; licensee Springer. This is an Open Access article distributed under the terms of the Creative Commons Attribution License (http://creativecommons.org/licenses/by/2.0), which permits unrestricted use, distribution, and reproduction in any medium, provided the original work is properly cited. 
for all $x \in \bar{\Omega}, \xi \in R^{N}, \nu \in R^{n N}$ and where $Q(x) \in L^{m, n-2 m+m \sigma}$ for some $\sigma>0$.

(H5) There exists $s$ with $s>n$ and a function $g \in H^{1, s}\left(\Omega, R^{N}\right)$ such that

$$
\left.u\right|_{\partial \Omega}=\left.g\right|_{\partial \Omega} .
$$

Note that we trivially have $g \in H^{1, m}\left(\Omega, R^{N}\right)$. Further, by the Sobolev embedding theorem, we have $g \in C^{0, \kappa}\left(\Omega, R^{N}\right)$ for any $\kappa \in\left[0,1-\frac{n}{s}\right]$. If $\left.g\right|_{\partial \Omega} \equiv 0$, we will take $g \equiv 0$ on $\Omega$.

If the domain in this case is a half unit ball, then the boundary condition is as follows:

$(\mathrm{H} 5)^{\prime}$ There exists $s$ with $s>n$ and a function $g \in H^{1, s}\left(B^{+}, R^{N}\right)$ such that

$$
\left.u\right|_{D}=\left.g\right|_{D}
$$

Here we write $B_{\rho}\left(x_{0}\right)=\left\{x \in R^{n}:\left|x-x_{0}\right|<\rho\right\}$, and further $B_{\rho}=B_{\rho}(0), B=B_{1}$. Similarly, we denote upper half balls as follows: for $x_{0} \in R^{n-1} \times\{0\}$, we write $B_{\rho}^{+}\left(x_{0}\right)$ for $\left\{x \in R^{n}: x_{n}>\right.$ $\left.0,\left|x-x_{0}\right|<\rho\right\}$ and set $B_{\rho}^{+}=B_{\rho}^{+}(0), B^{+}=B_{1}^{+}$. For $x_{0} \in R^{n-1} \times\{0\}$, we further write $D_{\rho}\left(x_{0}\right)$ for $\left\{x \in R^{n}: x_{n}=0,\left|x-x_{0}\right|<\rho\right\}$ and set $D_{\rho}=D_{\rho}(0), D=D_{1}$.

Definition 1.1 By a weak solution of (1.1), we mean for all test-functions $\varphi \in C_{0}^{1}\left(\bar{\Omega}, R^{N}\right)$, a function $u \in W^{1, m}\left(\bar{\Omega}, R^{N}\right)$ satisfying

$$
\int_{\Omega} A_{i j}^{\alpha \beta}(x, u)\left(D_{\beta} u^{j}, D_{\alpha} \varphi^{i}\right) d x=\int_{\Omega} B_{i}(x, u, D u) \cdot \varphi^{i} d x
$$

It is easily seen that by closure (1.2) holds for every $\varphi$ in $W^{1, m}\left(\bar{\Omega}, R^{N}\right)$.

Under such assumptions on the structural conditions, full regularity of (1.1) at the boundary cannot, in general, be expected [1] even if the boundary data is smooth. Then our goal is to establish a partial boundary regularity for weak solutions of systems (1.1).

There are some previous partial regularity results for quasilinear systems. For example, Arkhipova has studied regularity up to the boundary for nonlinear and quasilinear systems [2-4]. For systems in a diagonal form, boundary regularity was first established by Wiegner [5], and the proof was generalized and extended by Hildebrandt-Widman [6]. In the case of minima of functionals of the form $\int_{\Omega} A(x, u)|D u|^{2} d x$, Jost-Meier [7] established full regularity in a neighborhood of the boundary.

The result which is most closely related to that given here was shown by Grotowski [8]. Grotowski obtained boundary partial regularity results for more general systems: $-D_{\alpha} A_{i}^{\alpha}(\cdot, u, D u)=f^{i}(\cdot, u, D u), i=1, \ldots, N$. In this paper, we extend the results in [9]. However the results in the current paper do not follow from those in [8]; in the current situation, we need only to impose weaker structure conditions and, at the same time, we can obtain stronger conclusions.

The technique of $A$-harmonic approximation is a natural extension of the technique of harmonic approximation. This technique has its origins in Simon's [10] proof of the regularity theorem of Allard [11]. The technique of $A$-harmonic approximation then refers to the direct analog of the above situation. The interior version of this technique has previously been applied by Duzaar and Grotowski in [12].

In this context, as the argument for combining the boundary and the interior estimates is relatively standard, we omit it and obtain the following results. 
Theorem 1.1 Consider a bounded domain $\Omega$ in $R^{N}$, with a boundary of class $C^{1}$. Let $u$ be a bounded weak solution of (1.1) satisfying the boundary condition (H5), where the structure conditions (H1)-(H3) hold for $A_{i j}^{\alpha \beta}$, and (H4) holds for $B_{i}$. Consider a fixed $\gamma \in(0, \sigma]$. Then there exist positive $R_{1}$ and $\varepsilon_{0}$ (depending only on $n, N, \lambda, L, b, M, a(M), \omega(\cdot)$ and $\gamma$ ) with the property that

$$
f_{B_{R}\left(x_{0}\right) \cap \Omega}\left[\left|u-u_{x_{0}, R}^{\prime}\right|^{2}+\frac{\left|u-u_{x_{0}, R}^{\prime}\right|^{m}}{R^{m-2}}\right] d x+\left[\|g\|_{H^{1, s}}^{2}+\|g\|_{H^{1, s}}^{m}\right] R^{2\left(1-\frac{n}{s}\right)}+R^{2 \sigma} \leq \varepsilon_{0}^{2}
$$

for some $R \in\left(0, R_{1}\right]$ for a given $x_{0} \in \partial \Omega$ implies $u \in C^{0, \gamma}\left(\bar{B}_{\frac{R}{2}}\left(x_{0}\right) \cap \bar{\Omega}, R^{N}\right)$.

Note in particular that the boundary condition (H5) means that $u_{x_{0}, R}^{\prime}$ makes sense; in fact, we have $u_{x_{0}, R}^{\prime}=g_{x_{0}, R}^{\prime}$.

Combining this result with the analogous interior [13] and a standard covering argument allows us to obtain the following bound on the size of the singular set.

Corollary 1.1 Under the assumptions of Theorem 1.1, the singular set of the weak solution $u$ has $(n-m)$-dimensional Hausdorff measure zero in $\bar{\Omega}$.

If the domain of the main step in proving Theorem 1.1 is a half ball, the result is then:

Theorem 1.2 Consider a bounded weak solution of (1.1) on the upper half unit ball $B^{+}$ which satisfies the boundary condition $(\mathrm{H} 5)^{\prime}$, where the structure conditions $(\mathrm{H} 1)-(\mathrm{H} 3)$ hold for $A_{i j}^{\alpha \beta}$, and (H4) holds for $B_{i}$. Then there exist positive $R_{0}$ and $\varepsilon_{0}$ (depending only on $n, N$, $\lambda, L, b, M, a(M), M, \omega(\cdot)$ and $\gamma)$ with the property that

$$
f_{B_{R}^{+}\left(x_{0}\right)}\left[\left|u-u_{x_{0}, R}^{\prime}\right|^{2}+\frac{\left|u-u_{x_{0}, R}^{\prime}\right|^{m}}{R^{m-2}}\right] d x+\left[\|g\|_{H^{1, s}}^{2}+\|g\|_{H^{1, s}}^{m}\right] R^{2\left(1-\frac{n}{s}\right)}+R^{2 \sigma} \leq \varepsilon_{0}^{2}
$$

for some $R \in\left(0, R_{0}\right]$ for a given $x_{0} \in D$ implies that $u \in C^{0, \sigma}\left(\bar{B}_{\frac{R}{2}}^{+}\left(x_{0}\right), R^{N}\right)$ holds.

Note that analogously to above, the boundary condition (H5)' ensures that $u_{x_{0}, R}^{\prime}$ exists, and we have indeed $u_{x_{0}, R}^{\prime}=g_{x_{0}, R}^{\prime}$.

\section{The $A$-harmonic approximation technique}

In this section, we present the $A$-harmonic approximation lemma [9] and some standard results due to Campanato $[14,15]$.

Lemma 2.1 Consider fixed positive $\lambda$ and $L$, and $n, N \in N$ with $n \geq 2$. Then for any given $\varepsilon>0$, there exists $\delta=\delta(n, N, \lambda, L, \varepsilon) \in(0,1]$ with the following property: for any $A \in \operatorname{Bil}\left(R^{n N}\right)$ satisfying

$$
A(v, v) \geq \lambda|v|^{2} \quad \text { for all } v \in R^{n N}
$$

and

$$
|A(v, \bar{v})| \leq L|v||\bar{v}| \quad \text { for all } v, \bar{v} \in R^{n N},
$$


for any $w \in H^{1,2}\left(B_{\rho}^{+}\left(x_{0}\right), R^{N}\right)$ (for some $\left.\rho>0, x_{0} \in R^{n}\right)$ satisfying

$$
\rho^{2-n} \int_{B_{\rho}^{+}\left(x_{0}\right)}|D w|^{2} d x \leq 1
$$

and

$$
\left|\rho^{2-n} \int_{B_{\rho}^{+}\left(x_{0}\right)} A(D g, D \varphi) d x\right| \leq \delta \rho \sup _{B_{\rho}^{+}\left(x_{0}\right)}|D \varphi|
$$

and

$$
\left.w\right|_{D_{\rho}\left(x_{0}\right)}=0
$$

for all $\varphi \in C_{0}^{1}\left(B_{\rho}^{+}\left(x_{0}\right), R^{N}\right)$, there exists an A-harmonic function

$$
v \in \tilde{H}=\left\{\left.\tilde{w} \in H^{1,2}\left(B_{\rho}^{+}\left(x_{0}\right), R^{N}\right)\left|\rho^{2-n} \int_{B_{\rho}^{+}\left(x_{0}\right)}\right| D \tilde{w}\right|^{2} d x \leq 1,\left.\tilde{w}\right|_{D_{\rho}\left(x_{0}\right)} \equiv 0\right\}
$$

with

$$
\rho^{-n} \int_{B_{\rho}^{+}\left(x_{0}\right)}|v-w|^{2} d x \leq \varepsilon
$$

Next, we recall a slight modification of a characterization of Hölder continuous functions originally due to Campanato [14].

Lemma 2.2 Consider $n \in N, n \geq 2$ and $x_{0} \in R^{n-1} \times\{0\}$. Suppose that there are positive constants $\kappa$ and $\alpha$, with $\alpha \in(0,1]$, such that for some $v \in L^{2}\left(B_{6 R}^{+}\left(x_{0}\right)\right)$, the following inequalities hold:

$$
\inf _{\mu \in R}\left\{\rho^{-n} \int_{B_{\rho}^{+}(y)}|v-\mu|^{2} d x\right\} \leq \kappa^{2}\left(\frac{\rho}{R}\right)^{2 \alpha},
$$

for all $y \in D_{2 R}\left(x_{0}\right)$ and $\rho \leq 4 R$; and

$$
\inf _{\mu \in R} \rho^{-n}\left\{\int_{B_{\rho}(y)}|v-\mu|^{2} d x\right\} \leq \kappa^{2}\left(\frac{\rho}{R}\right)^{2 \alpha},
$$

for all $y \in B_{2 R}^{+}\left(x_{0}\right)$ and $B_{\rho}(y) \subset B_{2 R}^{+}\left(x_{0}\right)$.

Then there exists a Hölder continuous representative of the $L^{2}$-class of $v$ on $\bar{B}_{R}^{+}\left(x_{0}\right)$, and for this representative $\bar{v}$,

$$
|\bar{v}(x)-\bar{v}(z)| \leq C_{\kappa}\left(\frac{|x-z|}{R}\right)^{\alpha}
$$

holds for all $x, z \in \bar{B}_{R}^{+}\left(x_{0}\right)$, for a constant $C_{\kappa}$ depending only on $n$ and $\alpha$.

We close this section by a standard estimate for the solutions to homogeneous secondorder elliptic systems with constant coefficients due originally to Campanato [15]. 
Lemma 2.3 Consider fixed positive $\lambda$ and $L$, and $n, N \in N$ with $n \geq 2$. Then there exists $C_{0}$ depending only on $n, N$, $\lambda$ and $L$ (without loss of generality, we take $C_{0} \geq 1$ ) such that for $A \in \operatorname{Bil}\left(R^{n N}\right)$ satisfying (2.1) and (2.2), any A-harmonic function hon $B_{\rho}^{+}\left(x_{0}\right)$ with $\left.h\right|_{D_{\rho}\left(x_{0}\right)} \equiv$

0 satisfies

$$
\rho^{2} \sup _{B_{\frac{\rho}{2}}^{+}\left(x_{0}\right)}|D h|^{2} \leq C_{0} \rho^{2-n} \int_{B_{\rho}^{+}\left(x_{0}\right)}|D h|^{2} d x .
$$

\section{Caccioppoli inequality}

In this section, we prove the Caccioppoli inequality. First of all, we recall two useful inequalities. The Sobolev embedding theorem yields the existence of a constant $C_{s}$ depending only on $s, n$ and $N$ such that

$$
\sup _{B_{\rho}^{+}\left(x_{0}\right)}\left|g-g_{x_{0}, \rho}^{\prime}\right| \leq C_{s} \rho^{1-\frac{n}{s}}\|g\|_{H^{1, s}\left(B_{\rho}^{+}\left(x_{0}\right), R^{N}\right)}
$$

holds for $x_{0} \in D, \rho \leq 1-\left|x_{0}\right|$. Obviously, the inequality remains true if we replace $\|g\|_{H^{1, s}\left(B_{\rho}^{+}\left(x_{0}\right), R^{N}\right)}$ by $\|g\|_{H^{1, s}\left(B^{+}, R^{N}\right)}$, which we will henceforth abbreviate simply as $\|g\|_{H^{1, s}}$.

Now, we note that the Poincare inequality in this setting for $x_{0} \in D, \rho \leq 1-\left|x_{0}\right|$ yields

$$
\int_{B_{\rho}^{+}\left(x_{0}\right)}\left|g-g_{x_{0}, \rho}\right|^{m} d x \leq C_{p} \rho^{m} \int_{B_{\rho}^{+}\left(x_{0}\right)}|D g|^{m} d x
$$

for a constant $C_{p}$ which depends only on $n$.

Finally, we fix an exponent $\sigma \in(0,1)$ as follows: if $g \equiv 0, \sigma$ can be chosen arbitrarily (but henceforth fixed); otherwise, we take $\sigma$ fixed in $\left(0,1-\frac{n}{s}\right)$.

Then we prove an appropriate inequality for Caccioppoli.

Theorem 3.1 (Caccioppoli inequality) Let $u$ be a weak solution of systems (1.1) under (H1)(H5). Then there exists $\rho_{0}>0, \rho_{0}$, depending only on $L, M$, s and $\|g\|_{H^{1, s}}$, such that for all $B_{\rho}^{+}\left(x_{0}\right) \subset B^{+}$, with $x_{0} \in D^{+}, \rho<R<\rho_{0}$ there holds

$$
\begin{aligned}
& \int_{B_{\rho / 2}^{+}\left(x_{0}\right)}|D u|^{m} d x \\
& \leq C(\varepsilon, L) \int_{B_{\rho}^{+}\left(x_{0}\right)}\left[\frac{\left|u-u_{x_{0}, \rho}^{\prime}\right|^{2}}{\rho^{2}}+\frac{\left|u-u_{x_{0}, \rho}^{\prime}\right|^{m}}{\rho^{m}}\right] d x \\
& \quad+C(\varepsilon) \alpha_{n}\left[\rho^{n-2+2 \sigma}+\rho^{n-m+m \sigma}\right] \\
& \quad+C\left(\varepsilon, L, C_{s}, C_{p}\right)\left[\|g\|_{H^{1, s}}^{2}\left(\alpha_{n} \rho^{n} / 2\right)^{1-\frac{2}{s}}+\|g\|_{H^{1, s}}^{m}\left(\alpha_{n} \rho^{n} / 2\right)^{1-\frac{m}{s}}\right],
\end{aligned}
$$

where $C_{1}, C_{2}$ depending only on $\lambda$ and $L, m$ and $C_{3}$ depending on these quantities and, in addition, on $C_{s}, C_{p}$ and s.

Proof We consider a cut-off function $\eta \in C_{0}^{\infty}\left(B_{\rho}^{+}\left(x_{0}\right)\right)$ satisfying $0 \leq \eta \leq 1, \eta \equiv 1$ on $B_{\rho / 2}^{+}\left(x_{0}\right)$ and $|\nabla \eta|<\frac{4}{\rho}$. Then the function $(u-g) \eta^{2}$ is in $W_{0}^{1, m}\left(B_{\rho}^{+}\left(x_{0}, R^{N}\right)\right)$, and thus can 
be taken as a test-function in (1.2). By (H2) and (H4), we have

$$
\begin{aligned}
& \int_{B_{\rho}^{+}\left(x_{0}\right)} A_{i j}^{\alpha \beta}(\cdot, u)\left(D_{\beta} u^{j}, D_{\alpha} u^{i}\right) \eta^{2} d x \\
& \leq L \\
& \quad \int_{B_{\rho}^{+}\left(x_{0}\right)}\left(1+|u|^{2}\right)^{\frac{m-2}{2}}|D u||D g| \eta^{2} d x+2 \int_{B_{\rho}^{+}\left(x_{0}\right)}\left(1+|u|^{2}\right)^{\frac{m-2}{2}}|D u||D \eta| \eta|u-g| d x \\
& \quad+\int_{B_{\rho}^{+}\left(x_{0}\right)}\left(1+|u|^{2}\right)^{\frac{m-2}{2}}|Q(x)||u-g| \eta^{2} d x .
\end{aligned}
$$

Using (H5) and Young's inequality, we have

$$
\begin{aligned}
\int_{B_{\rho}^{+}\left(x_{0}\right)} & A_{i j}^{\alpha \beta}(\cdot, u)\left(D_{\beta} u^{j}, D_{\alpha} u^{i}\right) \eta^{2} d x \\
\leq & \frac{3 \varepsilon}{2} \int_{B_{\rho}^{+}\left(x_{0}\right)}\left(1+|u|^{2}\right)^{\frac{m-2}{2}}|D u|^{2} \eta^{2} d x+\frac{L^{2}}{2 \varepsilon} \int_{B_{\rho}^{+}\left(x_{0}\right)}\left(1+|u|^{2}\right)^{\frac{m-2}{2}}|D g|^{2} \eta^{2} d x \\
& +\frac{64 L^{2}+1}{2 \varepsilon \rho^{2}} \int_{B_{\rho}^{+}\left(x_{0}\right)}\left(1+|u|^{2}\right)^{\frac{m-2}{2}}\left|u-u_{x_{0}, \rho}^{\prime}\right|^{2} d x \\
& +\frac{64 L^{2}+1}{2 \varepsilon \rho^{2}} \int_{B_{\rho}^{+}\left(x_{0}\right)}\left(1+|u|^{2}\right)^{\frac{m-2}{2}}\left|g-g_{x_{0}, \rho}^{\prime}\right|^{2} d x \\
& +\varepsilon \int_{B_{\rho}^{+}\left(x_{0}\right)}\left(1+|u|^{2}\right)^{\frac{m-2}{2}} \rho^{2} \eta^{2}|Q(x)|^{2} d x \\
\leq & \frac{3 \varepsilon}{2} \int_{B_{\rho}^{+}\left(x_{0}\right)}\left(1+|u|^{2}\right)^{\frac{m-2}{2}}|D u|^{2} \eta^{2} d x \\
& +C(\varepsilon, L) \int_{B_{\rho}^{+}\left(x_{0}\right)}\left[\frac{\left|u-u_{x_{0}, \rho}^{\prime}\right|^{2}}{\rho^{2}}+\frac{\left|u-u_{x_{0}, \rho}^{\prime}\right|^{m}}{\rho^{m}}\right] d x \\
& +C(\varepsilon, L) \int_{B_{\rho}^{+}\left(x_{0}\right)}\left[|D g|^{2} \eta^{2}+|D g|^{m} \eta^{m}\right] d x \\
& +C(\varepsilon, L) \int_{B_{\rho}^{+}\left(x_{0}\right)}\left[\frac{\left|g-g_{x_{0}, \rho}^{\prime}\right|^{2}}{\rho^{2}}+\frac{\left|g-g_{x_{0}, \rho}^{\prime}\right|^{m}}{\rho^{m}}\right] d x \\
& +C(\varepsilon) \int_{B_{\rho}^{+}\left(x_{0}\right)}\left[\rho^{2} \eta^{2}|Q(x)|^{2}+\rho^{m} \eta^{m}|Q(x)|^{m}\right] d x .
\end{aligned}
$$

Recalling the definition of $Q(x)$ and by (3.1) and (3.2), we have

$$
\begin{aligned}
\int_{B_{\rho}^{+}\left(x_{0}\right)} & A_{i j}^{\alpha \beta}(\cdot, u)\left(D_{\beta} u^{j}, D_{\alpha} u^{i}\right) \eta^{2} d x \\
\leq & \frac{3 \varepsilon}{2} \int_{B_{\rho}^{+}\left(x_{0}\right)}\left(1+|u|^{2}\right)^{\frac{m-2}{2}}|D u|^{2} \eta^{2} d x \\
& +C(\varepsilon, L) \int_{B_{\rho}^{+}\left(x_{0}\right)}\left[\frac{\left|u-u_{x_{0}, \rho}^{\prime}\right|^{2}}{\rho^{2}}+\frac{\left|u-u_{x_{0}, \rho}^{\prime}\right|^{m}}{\rho^{m}}\right] d x \\
& +C\left(\varepsilon, L, C_{s}, C_{p}\right)\left[\|g\|_{H^{1, s}}^{2}\left(\alpha_{n} \rho^{n} / 2\right)^{1-\frac{2}{s}}+\|g\|_{H^{1, s}}^{m}\left(\alpha_{n} \rho^{n} / 2\right)^{1-\frac{m}{s}}\right] \\
& +C(\varepsilon) \alpha_{n}\left[\rho^{n-2+2 \sigma}+\rho^{n-m+m \sigma}\right] .
\end{aligned}
$$


Using (H2), we can get with $\varepsilon$ positive but arbitrary (to be fixed later)

$$
\begin{aligned}
(\lambda- & \left.\frac{3 \varepsilon}{2}\right) \int_{B_{R}^{+}\left(x_{0}\right)}\left(1+|u|^{2}\right)^{\frac{m-2}{2}}|D u|^{2} \eta^{2} d x \\
\leq & C(\varepsilon, L) \int_{B_{\rho}^{+}\left(x_{0}\right)}\left[\frac{\left|u-u_{x_{0}, \rho}^{\prime}\right|^{2}}{\rho^{2}}+\frac{\left|u-u_{x_{0}, \rho}^{\prime}\right|^{m}}{\rho^{m}}\right] d x+C(\varepsilon) \alpha_{n}\left[\rho^{n-2+2 \sigma}+\rho^{n-m+m \sigma}\right] \\
& +C\left(\varepsilon, L, C_{s}, C_{p}\right)\left[\|g\|_{H^{1, s}}^{2}\left(\alpha_{n} \rho^{n} / 2\right)^{1-\frac{2}{s}}+\|g\|_{H^{1, s}}^{m}\left(\alpha_{n} \rho^{n} / 2\right)^{1-\frac{m}{s}}\right] .
\end{aligned}
$$

We can find a constant $p=\min \left\{1,\left(\frac{1+|u|^{2}}{|D u|^{2}}\right)^{\frac{m-2}{2}}\right\}$ yields

$$
\begin{aligned}
(\lambda- & \left.\frac{3 \varepsilon}{2}\right) p \int_{B_{\rho / 2}^{+}\left(x_{0}\right)}|D u|^{m} d x \\
\leq & C(\varepsilon, L) \int_{B_{\rho}^{+}\left(x_{0}\right)}\left[\frac{\left|u-u_{x_{0}, \rho}^{\prime}\right|^{2}}{\rho^{2}}+\frac{\left|u-u_{x_{0}, \rho}^{\prime}\right|^{m}}{\rho^{m}}\right] d x+C(\varepsilon) \alpha_{n}\left[\rho^{n-2+2 \sigma}+\rho^{n-m+m \sigma}\right] \\
& +C\left(\varepsilon, L, C_{s}, C_{p}\right)\left[\|g\|_{H^{1, s}}^{2}\left(\alpha_{n} \rho^{n} / 2\right)^{1-\frac{2}{s}}+\|g\|_{H^{1, s}}^{m}\left(\alpha_{n} \rho^{n} / 2\right)^{1-\frac{m}{s}}\right] .
\end{aligned}
$$

Choosing $\varepsilon$ small enough, we can get the desired result immediately.

\section{The proof of the main theorem}

In this section, we proceed with the proof of the partial regularity result, and hence consider $u$ to be a weak solution of (1.1). We consider $x_{0} \in D$ and $y \in D_{R}\left(x_{0}\right), D_{\rho}(y) \subset \subset D_{R}\left(x_{0}\right)$, for $R<1-\left|x_{0}\right|$, and $\varphi \in C_{0}^{\infty}\left(B_{\frac{\rho}{2}}^{+}(y), R^{N}\right)$ with $\sup _{B_{\rho}^{+}(y)}|D \varphi| \leq 1$. First of all, we have

\section{Lemma 4.1}

$$
\int_{B_{\frac{\rho}{2}}^{+}(y)} A_{i j}^{\alpha \beta}\left(y, u_{y, \rho}^{\prime}\right)\left(D_{\beta} u^{j}, D_{\alpha} \varphi^{i}\right) d x \leq C_{4} \alpha_{n} \rho^{n-1} I^{\frac{1}{m}}\left(I^{\frac{m-1}{m}}+\omega(I)\right) \sup _{B_{\frac{\rho}{2}}^{+}\left(x_{0}\right)}|D \varphi|,
$$

here and hereafter, for $z \in D, r \in(0,1-|z|)$, we define

$$
I(z, r)=f_{B_{r}^{+}(z)}\left[\left|u-u_{z, r}^{\prime}\right|^{2}+\frac{\left|u-u_{z, r}^{\prime}\right|^{m}}{r^{m-2}}\right] d x+r^{2\left(1-\frac{s}{n}\right)}\left[\|g\|_{H^{1, s}}^{2}+\|g\|_{H^{1, s}}^{m}\right]+r^{2 \sigma} .
$$

Proof Consider $x_{0} \in D$ and $y \in D_{R}\left(x_{0}\right), D_{\rho}(y) \subset \subset D_{R}\left(x_{0}\right)$ for $R<1-\left|x_{0}\right|$, and $\varphi \in$ $C_{0}^{\infty}\left(B_{\frac{\rho}{2}}^{+}(y), R^{N}\right)$ with $\sup _{B_{\rho}^{+}(y)}|D \varphi| \leq 1$. Using (1.2), we have

$$
\begin{aligned}
& \int_{B_{\frac{\rho}{2}}^{+}(y)} A_{i j}^{\alpha \beta}\left(y, u_{y, \rho}^{\prime}\right)\left(D_{\beta} u^{j}, D_{\alpha} \varphi^{i}\right) d x \\
& \leq \int_{B_{\frac{\rho}{2}}^{+}(y)}\left(1+|u|^{2}\right)^{\frac{m-2}{2}}|Q(x)| \rho d x \sup _{B_{\frac{\rho}{2}}^{+}(y)}|D \varphi| \\
& \quad+\int_{B_{\frac{\rho}{2}}^{+}(y)}\left|A_{i j}^{\alpha \beta}(x, u)-A_{i j}^{\alpha \beta}\left(y, u_{y, \rho}^{\prime}\right)\right||D u| d x \sup _{B_{\frac{\rho}{2}}^{+}(y)}|D \varphi| .
\end{aligned}
$$


Henceforth, we restrict $\rho$ sufficiently small and keeping the definition of $Q(x)$ in mind,

$$
\begin{aligned}
& \int_{\frac{B_{\frac{\rho}{2}}^{+}(y)}{2}}\left(1+|u|^{2}\right)^{\frac{m-2}{2}}|Q(x)| d x \\
& \leq C(m, \varepsilon)\left[\int_{B_{\frac{\rho}{2}}^{+}(y)}|Q(x)| d x+\int_{B_{\frac{\rho}{2}}^{+}(y)} \frac{\left|u-u_{y, \rho}^{\prime}\right|^{m}}{\rho^{m}} d x+\int_{B_{\frac{\rho}{2}}^{+}(y)}|Q(x)|^{\frac{m}{2}} \rho^{\frac{m(m-2)}{2}}\right] d x \\
& \leq C(m, \varepsilon)\left[\alpha_{n} \rho^{n}\left(f_{B_{\frac{\rho}{2}}^{+}(y)}|Q(x)|^{m} d x\right)^{\frac{1}{m}}\left(f_{B_{\frac{\rho}{2}}^{+}(y)} d x\right)^{1-\frac{1}{m}}+\int_{B_{\frac{\rho}{2}}^{+}(y)} \frac{\left|u-u_{y, \rho}^{\prime}\right|^{m}}{\rho^{m}} d x\right. \\
& \left.\quad+\rho^{\frac{m(m-2)}{2}}\left(\int_{B_{\frac{\rho}{2}}^{+}(y)}|Q(x)|^{m} d x\right)^{\frac{1}{2}}\left(\int_{B_{\frac{\rho}{2}}^{+}(y)} d x\right)^{\frac{1}{2}}\right] \\
& \leq C(m, \varepsilon) \rho^{n-2}\left[C\left(\|Q(x)\|_{L^{m}}\right) \alpha_{n} \rho^{2}+f_{B_{\frac{\rho}{2}}^{+}(y)} \frac{\left|u-u_{y, \rho}^{\prime}\right|^{m}}{\rho^{m-2}} d x+\alpha_{n}^{\frac{1}{2}} \rho^{\frac{(m-2)^{2}+m+m \sigma}{2}}\right] \\
& \leq C\left(m, \varepsilon,\|Q(x)\|_{L^{m}}, \alpha_{n}\right) \rho^{n-2} I(y, \rho),
\end{aligned}
$$

where we define that for $z \in D, r \in(0,1-|z|)$,

$$
I(z, r)=f_{B_{r}^{+}(z)}\left[\left|u-u_{z, r}^{\prime}\right|^{2}+\frac{\left|u-u_{z, r}^{\prime}\right|^{m}}{r^{m-2}}\right] d x+r^{2\left(1-\frac{s}{n}\right)}\left[\|g\|_{H^{1, s}}^{2}+\|g\|_{H^{1, s}}^{m}\right]+r^{2 \sigma} .
$$

Applying (H3), Theorem 3.1 and Jensen's inequality, we can calculate from (4.2)

$$
\begin{aligned}
& \int_{\frac{B_{\frac{\rho}{2}}^{+}(y)}{}}\left|A_{i j}^{\alpha \beta}(x, u)-A_{i j}^{\alpha \beta}\left(y, u_{y, \rho}^{\prime}\right)\right||D u| d x \\
& \leq\left(\int_{B_{\rho}^{+}(y)}|D u|^{m} d x\right)^{\frac{1}{m}}\left(\int_{B_{\rho}^{+}(y)} \omega^{\frac{m}{m-1}}\left(\rho^{m}+\left|u-u_{y, \rho}^{\prime}\right|^{m}\right) d x\right)^{\frac{m-1}{m}} \\
& \leq C \alpha_{n} \rho^{n-1}\left(\rho^{m} f_{B_{\rho}^{+}(y)}\left[\frac{\left|u-u_{y, \rho}^{\prime}\right|^{2}}{\rho^{2}}+\frac{\left|u-u_{y, \rho}^{\prime}\right|^{m}}{\rho^{m}}\right] d x+\rho^{2 \sigma}\right. \\
& \left.\quad+\left[\|g\|_{H^{1, s}}^{2}+\|g\|_{H^{1, s}}^{m}\right] \rho^{2\left(1-\frac{n}{s}\right)}\right)^{\frac{1}{m}} \cdot \omega\left(\rho^{m}+f_{B_{\frac{\rho}{2}}^{+}(y)}\left|u-u_{y, \rho}^{\prime}\right|^{m} d x\right) \\
& \leq C \alpha_{n} \rho^{n-1} I^{\frac{1}{m}}(y, \rho) \omega(I(y, \rho)) .
\end{aligned}
$$

Take (4.3) and (4.4) into (4.2), for arbitrary $\varphi \in C_{0}^{\infty}\left(\Omega, R^{N}\right)$, we thus have

$$
\int_{B_{\frac{\rho}{2}}^{+}(y)} A_{i j}^{\alpha \beta}\left(y, u_{y, \rho}^{\prime}\right)\left(D_{\beta} u^{j}, D_{\alpha} \varphi^{i}\right) d x \leq C_{4} \alpha_{n} \rho^{n-1}\left[I+I^{\frac{1}{m}} \omega(I)\right] \sup _{B_{\frac{\rho}{2}}^{+}\left(x_{0}\right)}|D \varphi| .
$$

Multiplying through by $\left(\frac{\rho}{2}\right)^{2-n}$, this yields

$$
\left|\left(\frac{\rho}{2}\right)^{2-n} \int_{B_{\frac{\rho}{2}}^{+}(y)} A_{i j}^{\alpha \beta}\left(y, u_{y, \rho}^{\prime}\right)\left(D_{\beta} u^{j}, D_{\alpha} \varphi^{i}\right) d x\right| \leq C_{4} I^{\frac{1}{m}}\left[I^{\frac{m-1}{m}}+\omega(I)\right] \rho \sup _{B_{\frac{\rho}{2}}^{+}\left(x_{0}\right)}|D \varphi|,
$$

for $C_{4}$ defined by $C_{4}=C\left(m, \varepsilon, C_{1}, C_{2}, \alpha_{n}\right)$. 
Lemma 4.2 Consider $u$ satisfying the conditions of Theorem 1.1 and $\sigma$ fixed, then we can find $\delta$ and $s_{0}$ together with positive constants $C_{9}$ such that the smallness conditions

$$
0<\omega\left(s_{0}\right) \leq \frac{\delta}{2} \quad \text { and } \quad I\left(x_{0}, R\right) \leq C_{9}^{-1} \min \left\{\left(\frac{\delta}{2}\right)^{\frac{m}{m-1}}, s_{0}\right\}
$$

together imply the growth condition

$$
I(y, \theta \rho) \leq \theta^{2 \sigma} I(y, \rho) .
$$

Proof We now set $w=u-g$, using in turn (H1), Young's inequality and Hölder's inequality. We have from (4.5)

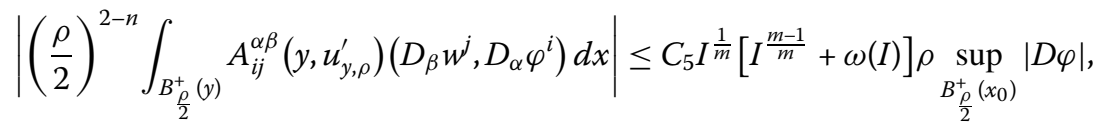

for $c_{5}=\max \left\{C_{4}, 2\left(\frac{\alpha_{n}}{2}\right)^{1-\frac{1}{s}}\right\}$.

We now define $C_{6}$ by $C_{6}=\max \left\{C_{5},\left[2^{2 n-1}\left(C_{1}+\alpha_{n}{ }^{-\frac{2}{s}} 2^{-(n+1)\left(1-\frac{2}{s}\right)}\right)\right]^{\frac{1}{2}}\right\}$ and set $v=\frac{w}{\gamma}$, for $\gamma=C_{6} I^{\frac{1}{m}}$. From (4.5), we then have

$$
\left|\left(\frac{\rho}{2}\right)^{2-n} \int_{B_{\frac{\rho}{2}}^{+}(y)} A_{i j}^{\alpha \beta}\left(y, u_{y, \rho}^{\prime}\right)\left(D_{\beta} v^{j}, D_{\alpha} \varphi^{i}\right) d x\right| \leq\left(I^{\frac{m-1}{m}}+\omega(I)\right) \rho \sup _{B_{\frac{\rho}{2}}^{+}\left(x_{0}\right)}|D \varphi|,
$$

and from (4.6), we observe from the definition of $C_{6}$ (recalling also the definition of $\gamma$ )

$$
\left(\frac{\rho}{2}\right)^{2-n} \int_{B_{\frac{\rho}{2}}^{+}(y)}|D v|^{2} d x<1
$$

Further, we note

$$
\left.v\right|_{D_{\rho}(y)}=\left.\frac{1}{\gamma} w\right|_{D_{\rho}(y)}=\left.\frac{1}{\gamma}(u-g)\right|_{D_{\rho}(y)} \equiv 0 .
$$

For $\varepsilon>0$, we take $\delta=\delta(n, N, \lambda, L, \varepsilon)$ to be the corresponding $\delta$ from $A$-harmonic approximation. Suppose that we could ensure that the smallness condition

$$
I^{\frac{m-1}{m}}+\omega(I) \leq \delta
$$

holds. Then in view of (4.7), (4.8) and (4.9), we would be able to apply Lemma 2.1 to conclude the existence of a function $h \in H^{1,2}\left(B_{\frac{\rho}{2}}^{+}(y), R^{N}\right)$ which is $A_{i j}^{\alpha \beta}\left(y, u_{y, \rho}^{\prime}\right)$-harmonic, with $\left.h\right|_{\frac{\rho}{2}(y)} \equiv 0$ such that

$$
\begin{aligned}
& \left(\frac{\rho}{2}\right)^{2-n} \int_{B_{\frac{\rho}{2}}^{+}(y)}|D h|^{2} d x \leq 1, \\
& \left(\frac{\rho}{2}\right)^{-n} \int_{B_{\frac{\rho}{2}}^{+}(y)}|v-h|^{2} d x \leq \varepsilon .
\end{aligned}
$$


For $\theta \in\left(0, \frac{1}{4}\right]$ arbitrary (to be fixed later), we have from the Campanato theorem, noting (4.11) and recalling also that $h(y)=0$,

$$
\sup _{B_{\theta \rho}^{+}(y)}|h|^{2} \leq \theta^{2} \rho^{2} \sup _{B_{\rho}^{+}(y)}|D h|^{2} \leq 4 C_{0} \theta^{2} .
$$

Using (4.12) and (4.13) we observe

$$
(\theta \rho)^{-n} \int_{B_{\theta \rho}^{+}(y)}|v|^{2} d x \leq 2(\theta \rho)^{-n}\left[\left(\frac{\rho}{2}\right)^{n} \varepsilon+\frac{1}{2} \alpha_{n}(\theta \rho)^{n} \sup _{B_{\theta \rho}^{+}(y)}|h|^{2}\right] \leq 2^{1-n} \theta^{-n} \varepsilon+4 \alpha_{n} C_{0} \theta^{2},
$$

and hence, on multiplying this through by $\gamma^{2}$, we obtain the estimate

$$
(\theta \rho)^{-n} \int_{B_{\theta \rho}^{+}(y)}|w|^{2} d x \leq C_{6}^{2}\left(2^{1-n} \theta^{-n} \varepsilon+4 \alpha_{n} C_{0} \theta^{2}\right) I^{\frac{2}{m}} .
$$

For the time being, we restrict to the case that $g$ does not vanish identically. Recalling that $w=u-g$, using in turn Poincare's, Sobolev's and then Hölder's inequalities, noting also that $u_{y, \theta \rho}^{\prime}=g_{y, \theta \rho}^{\prime}$, thus from (4.14), we can get

$$
\begin{aligned}
& (\theta \rho)^{-n} \int_{B_{\theta \rho}^{+}(y)}\left|u-u_{y, \theta \rho}^{\prime}\right|^{2} d x \\
& \leq 2(\theta \rho)^{-n}\left[\int_{B_{\theta \rho}^{+}(y)}|u-g|^{2} d x+\int_{B_{\theta \rho}^{+}(y)}\left|g-g_{y, \theta \rho}^{\prime}\right|^{2} d x\right] \\
& \quad \leq 2 C_{6}^{2}\left(2^{1-n} \theta^{-n} \varepsilon+4 \alpha_{n} C_{0} \theta^{2}\right) I^{\frac{2}{m}}+2 C_{p}(\theta \rho)^{2-n}\left[\frac{1}{2} \alpha_{n}(\theta \rho)^{n}\right]^{1-\frac{2}{s}}\|g\|_{H^{1, s}}^{2} \\
& \leq C_{7}\left(\theta^{-n} \varepsilon+\theta^{2}\right) I^{\frac{2}{m}}+C_{7} \theta^{2\left(1-\frac{n}{s}\right)} I
\end{aligned}
$$

for $C_{7}=\max \left\{8 \alpha_{n} C_{0} C_{6}^{2}, 2^{\frac{2}{s}} C_{p} \alpha_{n}^{1-\frac{2}{s}}\right\}$, and provided $\varepsilon=\theta^{n+2}$ and $\theta^{2} I^{\frac{2}{m}} \leq \theta^{2\left(1-\frac{n}{s}\right)} I$, we have

$$
(\theta \rho)^{-n} \int_{B_{\theta \rho}^{+}(y)}\left|u-u_{y, \theta \rho}^{\prime}\right|^{2} d x \leq 3 C_{7} \theta^{2\left(1-\frac{n}{s}\right)} I .
$$

At the same time, from (4.15), we can see that for $2<m<n(n \geq 3)$, we have $2<m<m$, where

$$
m^{*}=\left\{\begin{array}{ll}
\frac{m n}{n-m}, & \text { if } n>m, \\
m^{*}>m, & \text { if } m \geq n .
\end{array} \text { with } \frac{1}{m^{*}}<\frac{1}{m}<\frac{1}{2} .\right.
$$

Therefore, we can find $t \in[0,1]$ such that $\frac{1}{m}=\frac{1-t}{2}+\frac{t}{m^{2}}$.

Using Sobolev's, Caccioppoli's and Young's inequalities with (4.15), we have

$$
\begin{aligned}
& (\theta \rho)^{-n}(\theta \rho)^{-m+2} \int_{B_{\theta \rho}^{+}(y)}\left|u-u_{y, \theta \rho}^{\prime}\right|^{m} d x \\
& \leq \frac{1}{(\theta \rho)^{m-2}}\left((\theta \rho)^{-n} \int_{B_{\theta \rho}^{+}(y)}\left|u-u_{y, \theta \rho}^{\prime}\right|^{2} d x\right)^{\frac{(1-t) m}{2}}\left((\theta \rho)^{-n} \int_{B_{\theta \rho}^{+}(y)}\left|u-u_{y, \theta \rho}^{\prime}\right|^{m^{*}} d x\right)^{\frac{m t}{m^{*}}}
\end{aligned}
$$




$$
\begin{aligned}
& \leq \frac{1}{(\theta \rho)^{m-2}}\left(C_{7}\left(\theta^{-n} \varepsilon+\theta^{2}+\theta^{2\left(1-\frac{n}{s}\right)}\right) I^{\frac{2}{m}}\right)^{\frac{(1-t) m}{2}}\left((\theta \rho)^{m-n} \int_{B_{\theta \rho}^{+}(y)}|D u|^{m} d x\right)^{t} \\
& \leq\left(\frac{\alpha_{n}}{2}\right)^{t} C_{7}^{\frac{(1-t) m}{2}}\left[\left(\theta^{-n} \varepsilon+\theta^{2}+\theta^{2\left(1-\frac{n}{s}\right)}\right)\right]^{\frac{(1-t) m}{2}}\left[(\theta \rho)^{(m-2)(t-1)}\right] I \\
& \leq\left(\frac{\alpha_{n}}{2}\right)^{t} C_{7}^{\frac{(1-t) m}{2}}\left(\frac{(1-t) m}{2}\left(\theta^{-n} \varepsilon+\theta^{2}+\theta^{2\left(1-\frac{n}{s}\right)}\right)+\frac{m t}{m^{*}}(\theta \rho)^{\frac{(m-2)(t-1) m^{*}}{m t}}\right) I \\
& \leq C_{8}\left(\theta^{-n} \varepsilon+\theta^{2}+\theta^{2\left(1-\frac{n}{s}\right)}\right) I
\end{aligned}
$$

provided $(\theta \rho)^{\frac{(m-2)(1-t) m^{\prime \prime}}{m t}} \leq \theta^{2}$ and where $C_{8}=\left(\frac{\alpha_{n}}{2}\right)^{t} C_{7}^{\frac{(1-t) m}{2}}+1$.

We then fix $\varepsilon=\theta^{n+2}$, note that this also fixed $\delta$. Since $\rho \leq 1$, from the definition of $I$, we can get $\left[\|g\|_{H^{1, s}}^{2}+\|g\|_{H^{1, s}}^{m}\right](\theta \rho)^{2\left(1-\frac{n}{s}\right)} \leq \theta^{2\left(1-\frac{n}{s}\right)} I$, and $(\theta \rho)^{2 \sigma} \leq \theta^{2 \sigma} I$.

Combining these estimates with (4.16) and (4.17), we can get

$$
I(y, \theta \rho) \leq\left[3\left(C_{7}+C_{8}\right) \theta^{2\left(1-\frac{n}{s}\right)}+\theta^{2 \sigma}\right] I .
$$

Choosing $\theta \in\left(0, \frac{1}{4}\right]$ sufficiently small that there holds $3\left(C_{7}+C_{8}\right) \theta^{2\left(1-\frac{n}{s}\right)} \leq \theta^{2 \sigma}$, we can see from (4.18)

$$
I(y, \theta \rho) \leq \theta^{2 \sigma} I
$$

We now choose $s_{0}>0$ such that $0<\omega\left(s_{0}\right)<\left(\frac{\delta}{2}\right)$ and define $C_{9}$ by $C_{9}=\max \left\{2^{n+2 m-1}, 2 C_{6}^{2}+\right.$ $\left.1,2^{m-1} C_{s}^{m}+1\right\}$. Suppose that we have

$$
I\left(x_{0}, R\right) \leq C_{9}^{-1} \min \left\{\left(\frac{\delta}{2}\right)^{\frac{m}{m-1}}, s_{0}\right\}
$$

for some $R \in\left(0, R_{0}\right]$, where $R_{0}=\min \left\{\sqrt{2 s_{0}}, 1 / \sqrt{2 b}, 1-\left|x_{0}\right|\right\}$.

For any $y \in D_{\frac{R}{2}}\left(x_{0}\right)$, we use the Sobolev inequality to calculate

$$
\begin{aligned}
\frac{\alpha_{n} R^{n}}{2^{n+1}}\left|u_{x_{0}, R}^{\prime}-u_{y, \frac{R}{2}}^{\prime}\right|^{2} & \leq 2 \int_{B_{\frac{R}{2}}^{+}}\left|g-g_{x_{0}, R}^{\prime}\right|^{2} d x+2 \int_{B_{\frac{R}{2}}^{+}}\left|g-g_{y, \frac{R}{2}}^{\prime}\right|^{2} d x \\
& \leq 2 \alpha_{n} C_{s}^{2}\|g\|_{H^{1, s}}^{2} R^{n+2\left(1-\frac{n}{s}\right)},
\end{aligned}
$$

and

$$
\begin{aligned}
\frac{\alpha_{n} R^{n}}{2^{n+1}}\left|u_{x_{0}, R}^{\prime}-u_{y, \frac{R}{2}}^{\prime}\right|^{m} & \leq 2^{m-1} \int_{B_{\frac{R}{2}}^{+}}\left|g-g_{x_{0}, R}^{\prime}\right|^{m} d x+2^{m-1} \int_{B_{\frac{R}{2}}^{+}}\left|g-g_{y, \frac{R}{2}}^{\prime}\right|^{m} d x \\
& \leq 2^{m} \alpha_{n} C_{s}^{m}\|g\|_{H^{1, s}}^{m} R^{n+m\left(1-\frac{n}{s}\right)} .
\end{aligned}
$$

And then we can calculate

$$
\begin{aligned}
I\left(y, \frac{1}{2} R\right) \leq & 2^{n+2 m-1}\left[f_{B_{\frac{R}{2}}^{+}(y)}\left|u-u_{x_{0}, R}^{\prime}\right|^{2} d x+\frac{1}{R^{m-2}} f_{B_{\frac{R}{2}}^{+}(y)}\left|u-u_{x_{0}, R}^{\prime}\right|^{m} d x\right] \\
& +\left(2 C_{s}^{2}+1\right)\|g\|_{H^{1, s}}^{2} R^{2\left(1-\frac{n}{s}\right)}+\left(2^{m-1} C_{s}^{m}+1\right)\|g\|_{H^{1, s}}^{m} R^{2\left(1-\frac{n}{s}\right)}+\frac{1}{4} R^{2 \sigma} \\
\leq & C_{9} I\left(x_{0}, R\right) .
\end{aligned}
$$


Then we have

$$
I^{\frac{m-1}{m}}\left(y, \frac{1}{2} R\right)+\omega\left(I\left(y, \frac{1}{2} R\right)\right) \leq\left[C_{9} I\left(x_{0}, R\right)\right]^{\frac{m-1}{m}}+\omega\left(C_{9} I\left(x_{0}, R\right)\right) \leq \frac{1}{2} \delta+\omega\left(s_{0}\right) \leq \delta,
$$

which means that the condition (4.20) is sufficient to guarantee the smallness condition (4.10) for $\rho=\frac{R}{2}$, for all $y \in D_{\frac{R}{2}}\left(x_{0}\right)$. We can thus conclude that (4.18) holds in this situation. From (4.18), we thus have

$$
I^{\frac{m-1}{m}}(y, \theta \rho / 2)+\omega(I(y, \theta \rho / 2)) \leq I^{\frac{m-1}{m}}\left(y, \frac{1}{2} R\right)+\omega\left(I\left(y, \frac{1}{2} R\right)\right) \leq \delta,
$$

meaning that we can apply (4.18) on $B_{\theta \rho / 2}^{+}(y)$ as well, yielding

$$
I\left(y, \theta^{2} R / 2\right) \leq \theta^{4 \sigma} I(y, R / 2),
$$

and inductively,

$$
I\left(y, \theta^{k} R / 2\right) \leq \theta^{2 k \sigma} I(y, R / 2) .
$$

The next step is to go from a discrete to a continuous version of the decay estimate. Given $\rho \in(0, R / 2]$, we can find $k \in N_{0}$ such that $\theta^{k+1} R / 2<\rho \leq \theta^{k} R / 2$. We calculate in a similar manner to above. Firstly, we use the Sobolev inequality, to see

$$
\int_{B_{\rho}^{+}(y)}\left|u_{y, \rho}^{\prime}-u_{y, \theta^{k} R / 2}^{\prime}\right|^{2} d x \leq 2 \alpha_{n}\left(1 / 2 \theta^{k} R\right)^{n} C_{s}^{2}\|g\|_{H^{1, s}}^{2}\left(1 / 2 \theta^{k} R\right)^{2\left(1-\frac{n}{s}\right)}
$$

which allows us to deduce

$$
\int_{B_{\rho}^{+}(y)}\left|u-u_{y, \rho}^{\prime}\right|^{2} d x \leq 2 \int_{B_{\rho}^{+}(y)}\left|u-u_{y, \theta^{k} R / 2^{\prime}}\right|^{2} d x+4 \alpha_{n}\left(1 / 2 \theta^{k} R\right)^{n} C_{s}^{2}\|g\|_{H^{1, s}}^{2}\left(1 / 2 \theta^{k} R\right)^{2\left(1-\frac{n}{s}\right)},
$$

and similarly, we can get

$$
\int_{B_{\rho}^{+}(y)}\left|u_{y, \rho}^{\prime}-u_{y, \theta^{k} R / 2}^{\prime}\right|^{m} d x \leq 2^{m-1} \alpha_{n}\left(1 / 2 \theta^{k} R\right)^{n} C_{s}^{m}\|g\|_{H^{1, s}}^{m}\left(1 / 2 \theta^{k} R\right)^{m\left(1-\frac{n}{s}\right)},
$$

and

$$
\begin{aligned}
& \int_{B_{\rho}^{+}(y)}\left|u-u_{y, \rho}^{\prime}\right|^{m} d x \\
& \quad \leq 2^{m-1} \int_{B_{\rho}^{+}(y)}\left|u-u_{y, \theta^{k} R / 2}^{\prime}\right|^{m} d x+2^{2 m-2} \alpha_{n}\left(1 / 2 \theta^{k} R\right) C_{s}^{m}\|g\|_{H^{1, s}}^{m}\left(1 / 2 \theta^{k} R\right)^{m\left(1-\frac{n}{s}\right)}
\end{aligned}
$$

and hence, finally,

$$
I(y, \rho) \leq C_{10} I\left(y, \theta^{k} R / 2\right)
$$

for $C_{10}=8 \theta^{-n} C_{s}^{2}+2^{2 m-1} \theta^{-n} C_{s}^{m}+1$. Combining this with (4.22) and (4.21), we have

$$
I(y, \rho) \leq C_{10} \theta^{2 k \sigma} I(y, R / 2) \leq C_{10} C_{9} \theta^{-2 \sigma}\left(\frac{2 \rho}{R}\right)^{2 \sigma} I\left(x_{0}, R\right) \leq C_{10} C_{9}\left(\frac{2}{\theta}\right) I\left(x_{0}, R\right)\left(\frac{\rho}{R}\right)^{2 \sigma}
$$


and more particularly

$$
\inf _{\mu \in R^{N}} \int_{B_{\rho}^{+}(y)}|u-\mu|^{2} d x \leq C_{11} I\left(x_{0}, R\right)\left(\frac{\rho}{R}\right)^{2 \sigma}
$$

for $C_{11}$ given by $C_{11}=C_{10} C_{9}\left(\frac{2}{\theta}\right)^{2 \sigma}$. Recalling that this estimate is valid for all $y \in D$ and $\rho$ with $D_{\rho}(y) \subset D_{R / 2}\left(x_{0}\right)$, assuming only the condition (4.20) on $I\left(x_{0}, R\right)$. This yields - after replacing $R$ by $6 R$ - the boundary estimate (2.7) requiring to apply Lemma 2.2.

The analogous interior estimate to (4.22) is standard, and we obtain the desired interior estimate (2.8) by a standard argument. Hence, we can apply Lemma 2.2 and conclude the desired Hölder continuity.

\section{Competing interests}

The authors declare that they have no competing interests.

\section{Authors' contributions}

SC participated in design of the study and drafted the manuscript. ZT participated in conceived of the study and the amendment of the paper. All authors read and approved the final manuscript.

\section{Author details}

'Department of Mathematics and Information Science, Zhangzhou Normal University, Zhangzhou, Fujian 363000, China.

${ }^{2}$ School of Mathematical Science, Xiamen University, Xiamen, Fujian 361005, China.

\section{Acknowledgements}

This work was supported by the National Natural Science Foundation of China (Nos: 11201415, 11271305); the Natural Science Foundation of Zhejiang Province (Y61 10078); the Natural Science Foundation of Fujian Province (2012J01027) and Training Programme Foundation for Excellent Youth Researching Talents of Fujian's Universities (JA12205).

\section{Received: 5 May 2012 Accepted: 20 September 2012 Published: 3 October 2012}

\section{References}

1. Giaquinta, M: A counter-example to the boundary regularity of solutions to elliptic quasilinear systems. Manuscr. Math. 24, 217-220 (1978)

2. Arkhipova, AA: Regularity results for quasilinear elliptic systems with nonlinear boundary conditions. J. Math. Sci. 77(4), 3277-3294 (1995)

3. Arkhipova, AA: On the regularity of solutions of boundary-value problem for quasilinear elliptic systems with quadratic nonlinear. J. Math. Sci. 80(6), 2208-2225 (1995)

4. Arkhipova, AA: On the regularity of the oblique derivative problem for quasilinear elliptic systems. J. Math. Sci. 84(1), 817-822 (1997)

5. Wiegner, M: A Priori Schranken für Lösungen Gewisser Elliptischer Systeme. Manuscr. Math. 18, $279-297$ (1976)

6. Hildebrandt, S, Widman, KO: On the Hölder continuity of weak solutions of quasilinear elliptic systems of second order. Ann. Sc. Norm. Super. Pisa, Ser. IV 4, 144-178 (1977)

7. Jost, J, Meier, M: Boundary regularity for minima of certain quadratic functionals. Math. Ann. 262, 549-561 (1983)

8. Grotowski, JF: Boundary regularity for nonlinear elliptic systems. Calc. Var. Partial Differ. Equ. 15(3), 353-388 (2002)

9. Grotowski, JF: Boundary regularity for quasilinear elliptic systems. Commun. Partial Differ. Equ. 27(11-12), 2491-2512 (2002)

10. Simon, L: Lectures on Geometric Measure Theory. Australian National University Press, Canberra (1983)

11. Allard, WK: On the first variation of a varifold. Ann. Math. 95, 225-254 (1972)

12. Duzaar, F, Grotouski, JF: Partial regularity for nonlinear elliptic systems: the method of A-harmonic approximation. Manuscr. Math. 103, 267-298 (2000)

13. Giaquinta, M: Multiple Integrals in the Calculus of Variations and Nonlinear Elliptic Systems. Princeton University Press, Princeton (1983)

14. Campanato, S: Proprietà di Hölderianità di alcune classi di funzioni. Ann. Sc. Norm. Super. Pisa, Ser. III 17, 175-188 (1963)

15. Campanato, S: Equazioni ellittiche del //e ordine e spazi L $2, \lambda$. Ann. Mat. Pura Appl. 69, 321-381 (1965)

doi:10.1186/1029-242X-2012-220

Cite this article as: Chen and Tan: Boundary regularity for elliptic systems with supquadratic growth condition. Journal of Inequalities and Applications 2012 2012:220. 